Journal of Advanced Research in Fluid Mechanics and Thermal Sciences

Journal homepage: www.akademiabaru.com/arfmts.html ISSN: 2289-7879

\title{
The Approximation of the Solution of Wave Problems by Spectral Expansions Connected with Elliptic Differential Operators
}

\author{
Abdulkasim Akhmedov ${ }^{1,3,}{ }^{*}$, Mohd Zuki Salleh $^{1}$, Abdumalik Rakhimov $^{2}$ \\ Pusat Sains Matematik, Universiti Malaysia Pahang, Lebuhraya Tun Razak, 26300 Gambang, Kuantan, Pahang, Malaysia \\ Kulliyah of Engineering, International Islamic University of Malaysia, IIUM, 53100 Kuala Lumpur, Malaysia \\ Faculty of Mathematics and Science, Paragon International University, No 8, St 315, Boeng Kak 1, Toul Kork, 12151, Phnom Penh, Cambodia
}

\section{ARTICLE INFO ABSTRACT}

\section{Article history:}

Received 24 April 2021

Received in revised form 2 June 2021

Accepted 10 June 2021

Available online 27 August 2021

Keywords:

Spectral expansion; wave problem; elliptic differential operator

\begin{abstract}
The current research is devoted to the investigation of the spectral expansions of elliptic differential operators corresponding to singular distributions, which can be used to describe the vibration process made of thin elastic membrane stretched tightly over a circular frame. By using the property that deflection of the membrane during the motion remains small compared to the size of the membrane we have obtained the sufficient conditions for summability of the spectral expansions connected with wave problems on the regions of the rectangular type.
\end{abstract}

\section{Introduction}

In this paper, a numerical approximation of the solution of wave equations on the rectangular region is constructed based on Riesz means of the spectral expansions connected with elliptic differential operators. The studies of the spectral expansions of distributions in the spaces with the negative smoothness are carried out firstly, in the work of Alimov [1]. He obtained the precise conditions for localization of the spectral expansions of distributions in the Hilbert spaces, related to the Laplace operator. This work of Sh. Alimov gave impetus to conducting in-depth research of the spectral expansions of distributions, related to the differential operators in the development of the new methods of the investigation of the spectral expansions of distributions and in obtaining the final conditions of decomposability in different functional spaces with the negative smoothness. As continuation of the ideas of the work of Alimov [1], the convergence problems for spectral expansions of the distribution in the closed domain, connected with the Laplace operator was considered [2]. Another research by Rakhimov [3] has been carried out on the convergence of the spectral decompositions of the distributions in the weak topology. We note that the equiconvergence relation between index of the Bochner - Riesz means of the expansions and power of the singularity of the distributions with compact support in summation associated with the elliptic operator is

\footnotetext{
* Corresponding author.

E-mail address: aakhmedov@paragoniu.edu.kh
}

https://doi.org/10.37934/arfmts.86.2.101106 
studied by Fargana et al., [4], Rakhimov [5], Rakhimov et al., [6], and Fargana et al., [7]. The extension of the results on the convergence is discussed by Rakhimov [8,9]. The uniform convergence of the spectral expansions of the distributions connected with the Schrodinger operator is investigated by Ahmedov et al., [10]. We refer the readers to the research by Ahmedov et al., [11] for the results on the convergence of the eigenfunction expansions of the distributions on the unit sphere. The problems on the generalized localization of the spectral expansions of the distributions are considered in the papers by Alimov [12] and Ashurov et al., [13]. A survey of all the techniques related to contrast enhancement of images is given in [14], which also contains all the advantages and disadvantages of these techniques.

In this paper, we study the vibration problem made of thin elastic membrane stretched tightly over a rectangular frame. The deflection of the membrane during the motion is small compared to the size of the membrane. Solution of wave transfer problems are subjected to the boundary conditions and initial conditions and had a form of double Fourier series. We investigated the spectral expansions of elliptic differential operators corresponding to singular distributions, which can be used to describe the vibration process made of thin elastic membrane stretched tightly over a circular frame. By using the property that deflection of the membrane during the motion remains small compared to the size of the membrane we have obtained the sufficient conditions for summability of the spectral expansions connected with wave problems on the rectangular plate.

\section{Formulation}

We consider a rectangular plate whose length and width are equal to $\pi$, say $\mathrm{R}=\{(x, y): 0 \leq x \leq$ $\pi, 0 \leq y \leq \pi\}$. Let $C_{0}^{\infty}(R)$ denotes the space of infinite times differentiable functions with compact support in $R$. A distribution $u$ in $R$ is a linear functional on $C_{0}^{\infty}(R)$ such that for every compact set $\mathcal{K} \subset R$ there exist constants $c$ and $k$ such that

$$
|u(\phi)| \leq c \sum_{\alpha+\beta \leq k} \sup _{x \in \mathcal{K}}\left|\frac{\partial^{\alpha+\beta}}{\partial x^{\alpha} \partial y^{\beta}} \phi(x, y)\right|, \phi(x, y) \in C_{0}^{\infty}(\mathcal{K})
$$

We denote a set of all distributions in $R$ by $\mathfrak{D}^{\prime}(R)$. That $u$ is linear functional on $C_{0}^{\infty}(R)$, then for the complex numbers $a, b$ we have

$u(a \cdot \phi+b \cdot \psi)=a \cdot u(\phi)+b \cdot u(\psi)$

for all $\phi, \psi \in C_{0}^{\infty}(R)$. One defines the Dirac measure $\delta$ by

$\delta(\phi)=\phi(0,0)$

Several other properties of the Dirac delta function $\delta(x, y)$ follow from its definition. In particular we have

$\delta(-x,-y)=\delta(x, y)$

Let proceed with the establishment of the mathematical model for the vibration process over the rectangular region. It is assumed that the displacements of each point on a rectangular plate $R$ are small and that the motion is such that each point on the plate moves in a direction perpendicular to the $x y$-plane (transverse vibration) - that is, the $u$-axis is perpendicular to the $x y$-plane. A physical 
model to visualize this phenomenon while working through this work is a vibrating drumhead of rectangular shape which is clamped along its frame. Let $u(x, y, t)$ denote the vertical displacement of any point on the rectangular plate $R$ measured from the $x y$-plane for $t>0$. We further assume that the material of the plate is perfectly flexible and it is homogenous (its mass per unit area $\rho$ is a constant), the displacement which is represented by $u$ is small in comparison to the area of the plate, the slope of the surface is small at all points, the tension $\mathbf{T}$ acts tangent to the plate, and its magnitude $\mathrm{T}$ is the same at all points, the tension is large compared with the force of gravity and no other external forces act on the plate. Moreover, for simplicity, we denote $a^{2}=T / \rho, a>0$ since both T and $\rho$ are positive quantities. Then, the mathematical model of the wave problem to be considered is as follows:

$$
\frac{\partial^{2} u}{\partial t^{2}}=a^{2}\left(\frac{\partial^{2} u}{\partial x^{2}}+\frac{\partial^{2} u}{\partial y^{2}}\right), 0<x<\pi, 0<y<\pi, t>0
$$

subject to the following boundary conditions

$$
\begin{aligned}
& u(0, y, t)=0, u(\pi, y, t)=0, t>0 \\
& u(x, 0, t)=0, u(x, \pi, t)=0, t>0
\end{aligned}
$$

And subject to the following initial conditions, as well

$$
\begin{aligned}
& u(x, y, 0)=0 \\
& \left.\frac{\partial u}{\partial t}\right|_{t=0}=\delta\left(x-\frac{\pi}{2}, y-\frac{\pi}{2}\right), 0<x<\pi, 0<y<\pi
\end{aligned}
$$

\section{Results and Discussions}

By combining all the results obtained above, we find that for an arbitrary pair of positive integers $m$ and $n$, we have the normal mode

$$
u_{m n}(x, y, t)=X_{m}(x) Y_{n}(y) T_{m n}(t)=\sin \mu_{m} x \sin \eta_{n} y\left(b_{m n} \sin \left(\lambda_{m n} t\right)\right)
$$

For any choice of constants $A_{m n}$ and $B_{m n}$, by the principle of superposition we conclude that the general solution that satisfy both the heat Eq. (1) and boundary conditions (2) is

$$
u(x, y, t)=\sum_{m=1}^{\infty} \sum_{n=1}^{\infty} B_{m n} \sin \mu_{m} x \sin \eta_{n} y \sin \left(\lambda_{m n} t\right)
$$

Now, we need to determine the value of $B_{m n}$. First, we differentiate Eq. (4) with respect to $t$ and obtain

$$
\frac{\partial u}{\partial t}=\sum_{m=1}^{\infty} \sum_{n=1}^{\infty} \lambda_{m n} \cos \left(\lambda_{m n} t\right) \sin \mu_{m} x \sin \eta_{n} y
$$

Setting $t=0$ in the latter and using the initial conditions in Eq. (3), we get 


$$
\left.\frac{\partial u}{\partial t}\right|_{t=0}=\sum_{m=1}^{\infty} \sum_{n=1}^{\infty} B_{m n} \lambda_{m n} \sin \mu_{m} x \sin \eta_{n} y=\delta\left(x-\frac{\pi}{2}, y-\frac{\pi}{2}\right)
$$

From the last equation, we determine $B_{m n}$ as follows

$$
B_{m n} \lambda_{m n}=\left(\frac{2}{\pi}\right)^{2} \int_{0}^{\pi} \int_{0}^{\pi} \delta\left(x-\frac{\pi}{2}, y-\frac{\pi}{2}\right) \cdot\left(\sin \mu_{m} x \sin \eta_{n} x\right) d x d y
$$

We can evaluate the above double-integral using the following property of Dirac delta function

$$
\int_{-\infty}^{\infty} \int_{-\infty}^{\infty} f(x, y) \delta(x-a, y-b) d x d y=f(a, b)
$$

Then, we obtain

$$
B_{m n}=\left(\frac{2}{\pi}\right)^{2} \frac{1}{\lambda_{m n}} \sin \frac{\mu_{m} \pi}{2} \sin \frac{\eta_{n} \pi}{2}
$$

where $\mu_{m}=m, \eta_{n}=n, \lambda_{m n}=a \sqrt{\mu_{m}^{2}+\eta_{n}^{2}}$, and $B_{m n}=\left(\frac{2}{\pi}\right)^{2} \frac{1}{\lambda_{m n}} \sin \frac{\mu_{m} \pi}{2} \sin \frac{\eta_{n} \pi}{2}$.

Finally, by substituting the obtained values to Eq. (4) we obtain the solution of the wave problem (1) which satisfies the given boundary conditions (2) and initial conditions (3)

$$
u(x, y, t)=\left(\frac{2}{\pi}\right)^{2} \sum_{n=1}^{\infty} \sum_{m=1}^{\infty} \frac{\sin \frac{m \pi}{2} \sin \frac{n \pi}{2}}{\sqrt{n^{2}+m^{2}}} \sin \left(\sqrt{m^{2}+n^{2}} t\right) \sin m x \sin n y
$$

We note here that Dirac delta function is participating in the initial conditions for the $u_{t}(x, y, t)$ and we investigate the sufficient conditions which guarantees the convergence of the following series

$$
\left(\frac{2}{\pi}\right)^{2} \sum_{n=1}^{\infty} \sum_{m=1}^{\infty} \sin \frac{m \pi}{2} \sin \frac{n \pi}{2} \cos \left(\sqrt{m^{2}+n^{2}} t\right) \sin m x \sin n y
$$

The latter series is obtained from formal differentiation of the Eq. (5), which converges in the topology of distributions to $u_{t}(x, y, t)$. Let define the Riesz means of order $s$ of the expansions of $u_{t}(x, y, t)$ by the following

$$
E_{\lambda}^{S} u_{t}(x, y, t)=\left(\frac{2}{\pi}\right)^{2} \sum_{\sqrt{m^{2}+n^{2}}<\lambda}\left(1-\frac{\sqrt{n^{2}+m^{2}}}{\lambda}\right)^{S} \sin \frac{m \pi}{2} \sin \frac{n \pi}{2} \cos \left(\sqrt{m^{2}+n^{2}} t\right) \sin m x \sin n y
$$

Next, we check whether the Riesz means $E_{\lambda}^{s} u_{t}(x, y, t)$ approximate the function $u_{t}(x, y, t)$ for different orders of Riesz means.

The numerical approximations displayed in Table 1 show that the Fourier series diverges for $\mathrm{s}=$ 0 . After regularization the Reisz means of order $s=1$, the Fourier series also diverges. Finally, for $s=$ 
2 the series converges. The solution of the Riesz means of order $s=2$ of the Fourier series is approximately equal to zero.

\section{Table 1}

Regularized solution of heat equation for different values of $s(s=0, s=1, s=2)$, at the point $x=\frac{\pi}{4}, y=\frac{\pi}{4}$ and $t=0$

\begin{tabular}{|c|c|c|c|}
\hline \multicolumn{4}{|c|}{ Result from MATLAB } \\
\hline$\lambda$ & $E_{\lambda}^{0} u_{t}(\pi / 4, \pi / 4,0)$ & $E_{\lambda}^{1} u_{t}(\pi / 4, \pi / 4,0)$ & $E_{\lambda}^{2} u_{t}(\pi / 4, \pi / 4,0)$ \\
\hline 850 & 7.00000000000111 & -0.00411526008122465 & 0.00315287084334459 \\
\hline 2000 & $7.05258074162887 \mathrm{e}-12$ & 0.00437619755418917 & 0.00224139519878630 \\
\hline 2500 & -5.99999999999709 & -0.00424875153686566 & 0.00139881698091759 \\
\hline 3500 & -11.99999999999978 & 0.00265330270521225 & 0.000670850331949691 \\
\hline 4000 & -1.499999999998671 & -0.00301893616894222 & 0.000496940178414063 \\
\hline 6000 & -14.49999999999866 & 0.00390290242268027 & 0.000192221516156804 \\
\hline 6500 & 8.00000000000999 & -0.00459805620623655 & 0.000158396465790134 \\
\hline 7000 & 4.50000000002167 & 0.00370161678701597 & 0.000129784596000590 \\
\hline 8000 & 17.5000000000110 & -0.00293904530899636 & 0.000091956257842550 \\
\hline 8500 & -21.99999999999889 & 0.00475047959718943 & 0.000077734281354600 \\
\hline 9000 & 9.99999999997983 & -0.00392974892915876 & 0.000066921895074356 \\
\hline
\end{tabular}

As it is expected, the numerical date displayed in Table 2 verifies that after critical point, the Reisz means of the Fourier series converges and below critical point the Reisz means of the Fourier series diverges. Although the numerical approximation of the series is very close to zero at critical points, it still diverges.

\section{Table 2}

Regularized solution of heat equation for different values of $s(s=1.4, s=1.5, s=1.6), x=\frac{\pi}{4}$, $y=\frac{\pi}{4}$ and $t=0$

\begin{tabular}{|c|c|c|c|}
\hline \multicolumn{4}{|c|}{ Result from MATLAB } \\
\hline$\lambda$ & $E_{\lambda}^{1.4} \frac{\partial}{\partial t} u(\pi / 4, \pi / 4,0)$ & $E_{\lambda}^{1.5} \frac{\partial}{\partial t} u(\pi / 4, \pi / 4,0)$ & $E_{\lambda}^{1.6} \frac{\partial}{\partial t} u(\pi / 4, \pi / 4,0)$ \\
\hline 850 & 0.00803593046444506 & -0.00115524562312308 & 0.00142023240665223 \\
\hline 2000 & 0.000875761946008247 & 0.000963512443736381 & 0.00112013058002347 \\
\hline 2500 & -0.000784349026601738 & -0.000780214420703640 & 0.000834852932308561 \\
\hline 3500 & -0.000499871318010436 & 0.000780214420703640 & 0.000476428457911737 \\
\hline 4000 & -0.00204231619672313 & -0.000286399500720402 & 0.00033969719526239 \\
\hline 6000 & -0.000187415490236209 & 0.000177104174169329 & 0.000175328908221639 \\
\hline 6500 & 0.000152049739436416 & -0.000152735642328927 & 0.000152411243847878 \\
\hline 7000 & 0.000248924682223761 & 0.000767207453907792 & 0.000102762495466868 \\
\hline 8000 & 0.00508716709242962 & -0.000739754672066731 & 0.0000843421787644696 \\
\hline 8500 & -0.000859976145705169 & 0.000796355406602819 & 0.0000775983948437184 \\
\hline 9000 & 0.000776983513026165 & -0.000771428936626843 & 0.0000731753987851646 \\
\hline
\end{tabular}

\section{Conclusion}

The solution of the vibration problems had a form of double Fourier series and it requires some regularizations. Based on the singularity, we considered the Reisz method of summation as regularization of the Fourier series solutions of the wave problems. To minimize the length of numerical calculations of the wave problems, we optimize the regularized Fourier series solutions. To optimize the regularization of the solutions of the plate vibration problems we found the minimum order of the Reisz means. The minimum order was $s>(N-1) / 2-I$. Therefore, a programming 
(MATLAB) is used for the numerical solutions. We found the optimization of the regularization of the series solutions at a fixed point of the plates at initial time and critical index. As it is estimated, we achieved the good convergence after critical point. Moreover, we studied optimization of the regularization of the Fourier series of rectangular plate vibration problem and a numerical method is used to find the series solution at a fixed point of the plate at initial time and critical index. Numerical approximations of the solution of the wave equation are constructed to verify the convergence theorem.

\section{Acknowledgement}

This paper is supported by Universiti Malaysia Pahang under Research Scheme RDU190356.

\section{References}

[1] Alimov Sh, A. "On the spectral decompositions of distributions." In Doklady Mathematics, vol. 331, pp. 661-662. 1993.

[2] Alimov, S. A., and A. A. Rakhimov. "Localization of spectral expansions of distributions." Differential Equations 32, no. 6 (1996): 798-802.

[3] Rakhimov, Abdumalik A. "On The Approximation of The Spectral Expansions of Distributions in The Weak Topology." Australian Journal of Basic and Applied Sciences 6, no. 2 (2012): 20-24.

[4] Fargana, A., A. A. Rakhimov, A. A. Khan, and T. B. H. Hassan. "Equiconvergence in Summation Associated with Elliptic Polynomial." In Journal of Physics: Conference Series, vol. 949, no. 1, p. 012001. IOP Publishing, 2017. https://doi.org/10.1088/1742-6596/949/1/012001

[5] Rakhimov, A. A. "On the equiconvergence of the Fourier series and the Fourier integral of distributions." In AIP Conference Proceedings, vol. 1739, no. 1, p. 020060. AIP Publishing LLC, 2016. https://doi.org/10.1063/1.4952540

[6] Rakhimov, Abdumalik A., Torla Bin Hj Hassan, and Ahmad Fadly Nurullah bin Rasedee. "On equiconvergence of Fourier series and Fourier integral." In Journal of Physics: Conference Series, vol. 819, no. 1, p. 012025. IOP Publishing, 2017. https://doi.org/10.1088/1742-6596/819/1/012025

[7] Fargana, A., A. A. Rakhimov, A. A. Khan, and T. B. H. Hassan. "Optimization of the Regularization of the Solution of the Plate Vibration Problem." International Journal of Applied Engineering Research 13, no. 8 (2018): 6364-6368.

[8] Rakhimov, A. "On the summability of the spectral expansions associated with the elliptic differential operators." In Journal of Physics: Conference Series, vol. 1132, no. 1, p. 012013. IOP Publishing, 2018. https://doi.org/10.1088/1742-6596/1132/1/012013

[9] Rakhimov, Abdumalik. "Localization of the Spectral Expansions Associated with the Partial Differential Operators." In Mathematical Methods in Engineering, pp. 217-233. Springer, Cham, 2019. https://doi.org/10.1007/978-3-31991065-9 11

[10] Ahmedov, Anvarjon A., Nur Amalina Binti Jamaludin, and Abdumalik Rakhimov. "Uniformly Convergence of The Spectral Expansions of The Schrödinger Operator on A Closed Domain." In Journal of Physics: Conference Series, vol. 435, no. 1, p. 012014. IOP Publishing, 2013. https://doi.org/10.1088/1742-6596/435/1/012014

[11] Ahmedov, Anvarjon A., Ahmad Fadly Nurullah bin Rasedee, and Abdumalik Rakhimov. "On the sufficient conditions of the localization of the Fourier-Laplace series of distributions from liouville classes." In Journal of Physics: Conference Series, vol. 435, no. 1, p. 012016. IOP Publishing, 2013. https://doi.org/10.1088/17426596/435/1/012016

[12] Alimov, Sh A. "Generalized localization of Riesz means of spectral expansions of distributions." In Doklady Mathematics, vol. 86, no. 2, pp. 597-599. SP MAIK Nauka/Interperiodica, 2012. https://doi.org/10.1134/S1064562412050018

[13] Ashurov, Ravshan, Almaz Butaev, and Biswajeet Pradhan. "On generalized localization of Fourier inversion associated with an elliptic operator for distributions." In Abstract and Applied Analysis, vol. 2012. Hindawi, 2012. https://doi.org/10.1155/2012/649848

[14] Mustafa, Wan Azani, Haniza Yazid, and Noratikah Mazlan. "An enhancement method on illumination images: A survey." Journal of Advanced Review on Scientific Research 28, no. 1 (2016): 33-41. 